\title{
Carbohydrate intake and short-term regulation of leptin in humans
}

\author{
A. B.Jenkins ${ }^{1}$, T. P. M arkovic ${ }^{2}$, A . Fleury' ${ }^{2}$, L.V.C ampbell ${ }^{2}$ \\ ${ }^{1}$ Metabolic Research Centre and Department of Biomedical Science, University of Wollongong, Wollongong, Australia \\ ${ }^{2}$ Garvan Institute of Medical Research, St. Vincent's Hospital, Sydney, Australia
}

Summary The response of serum leptin to short (4 days) and prolonged (28 days) energy restriction (50\% reduction in energy intake) was determined in 18 (9 male, 9 female) moderately obese humans (body mass index $32.0 \pm 0.6 \mathrm{~kg} / \mathrm{m}^{2}$ mean $\pm \mathrm{SEM}$ ), 9 of whom had mild non-insulin-dependent diabetes mellitus (NIDDM). Body composition was assessed before and at the end of the energy restriction using DEXA. The subjects lost a measured $2.6 \pm 0.4 \mathrm{~kg}$ of body fat after 28 days and an estimated $0.3 \mathrm{~kg}$ at 4 days. Serum leptin fell to $64 \pm 3 \%$ of baseline levels at day 4 and further to $46 \pm 4 \%$ at day 28 . In a multiple correlation analysis, the change in leptin concentration at day 4 was significantly related to the change in dietary carbohydrate intake (partial $r=0.68$, $p<0.005)$ but not to changes in fat $(r=0.12)$ or protein $(r=0.02)$ intakes. There was a $1: 1$ relationship between the changes in leptin and dietary carbohydrate (regression slope $=1.0 \pm 0.3$ ). Gender, or the presence of NIDDM had no effects on these responses. This pronounced fall in serum leptin in association with reduced carbohydrate intake before substantial loss of body fat suggests a role for leptin in defending the body's carbohydrate stores and implicates leptin in the satiating effects of carbohydrate. Dietary or other interventions which maintain leptin levels during weight reduction may lead to improvements in weight loss. [Diabetologia (1997) 40: 348351]

Keywords Leptin, obesity, energy restriction, diabetes mellitus, non-insulin-dependent.
Leptin expression and secretion are strongly correlated with body fat mass in animals and humans in cross-sectional studies, and experimental alterations in body fat content are associated with parallel changes in leptin expression and secretion $[1,2]$. Leptin expression and secretion in animals is also acutely influenced by food restriction and refeeding $[2,3]$. In obese humans and animals undergoing energy restriction, amelioration of many of the metabolic abnormalities associated with obesity is apparent before

Received: 29 October 1996 and in revised form: 16December 1996

Corresponding author: A. B. Jenkins PhD, Department of Biomedical Science, University of Wollongong, Northfields Avenue, Wollongong, NSW Australia 2522

A bbreviations: NIDDM, Non-insulin-dependent diabetes mellitus. any major effect on body fat content. In an investigation of the mechanisms responsible for these early effects we have studied metabolic responses after 4 and 28 days of energy restriction in a group of moderately obese human subjects with and without non-insulindependent diabetes mellitus (NIDDM). We report here the responses of circulating leptin concentrations under these conditions, and their relationships to changes in macronutrient intakes and body composition.

\section{Subjects and methods}

Subjects: Nine (5 female, 4 male) obese subjects (BMI $=31.6 \pm 0.8($ mean \pm SEM $\left.) \mathrm{kg} / \mathrm{m}^{2}\right)$ with normal glucose tolerance and no family history of NIDDM, and 9 (4 female, 5 male) obese subjects $(\mathrm{BMI}=32.3 \pm 0.9)$ with mild NIDDM (fasting plasma glucose $\leq 12 \mathrm{mmol} / \mathrm{l}$ ), matched for age and 
Table 1. Macronutrient and total energy intakes before and during energy restriction in 18 moderately obese subjects

\begin{tabular}{|c|c|c|c|c|c|}
\hline \multirow[t]{2}{*}{ Macronutrients } & \multicolumn{2}{|l|}{ Pre-diet } & \multicolumn{2}{|l|}{ Diet } & \multirow{2}{*}{$\begin{array}{l}\text { Diet/Pre-diet } \\
(\%)(B / A \times 100)\end{array}$} \\
\hline & (g or MJ/day) (A) & (\% of energy) & (g or $\mathrm{MJ} /$ day) $(\mathrm{B})$ & (\% of energy) & \\
\hline $\begin{array}{l}\text { Carbohydrate } \\
\text { (g/day) }\end{array}$ & $\begin{array}{l}223 \pm 15 \\
(141-376)\end{array}$ & $\begin{array}{l}38.5 \pm 1.9 \\
(19-49)\end{array}$ & $\begin{array}{l}106 \pm 5^{\mathrm{b}} \\
(69-158)\end{array}$ & $\begin{array}{l}38.3 \pm 0.8 \\
(29-44)\end{array}$ & $\begin{array}{l}49 \pm 2 \\
(36-71)\end{array}$ \\
\hline $\begin{array}{l}\text { Fat } \\
\text { (g/day) }\end{array}$ & $\begin{array}{l}95 \pm 8 \\
(38-157)\end{array}$ & $\begin{array}{l}35.8 \pm 1.3 \\
(27-43)\end{array}$ & $\begin{array}{l}34 \pm 2^{\mathrm{b}} \\
(17-44)\end{array}$ & $\begin{array}{l}28.1 \pm 0.8^{b} \\
(22-35)\end{array}$ & $\begin{array}{l}40 \pm 3 \\
(21-74)\end{array}$ \\
\hline $\begin{array}{l}\text { Protein } \\
\text { (g/day) }\end{array}$ & $\begin{array}{l}109 \pm 9 \\
(65-188)\end{array}$ & $\begin{array}{l}19.3 \pm 0.8 \\
(12-25)\end{array}$ & $\begin{array}{l}84 \pm 4^{\mathrm{b}} \\
(54-123)\end{array}$ & $\begin{array}{l}32.3 \pm 0.9^{b} \\
(21-36)\end{array}$ & $\begin{array}{l}83 \pm 5 \\
(40-119)\end{array}$ \\
\hline
\end{tabular}

Mean \pm SEM (range)

${ }^{a}$ Sum of the tabulated macronutrient intakes + alcohol intake (pre-diet $6.4 \%$, diet $1.3 \%$ )

anthropometry, were recruited from a diabetes clinic and through advertisements in the press. Subjects had been weight stable for at least 6 months prior to commencement of the study. Subjects with NIDDM were either treated with diet $(n=7)$ or small doses of oral medication (metformin $n=1$, gliclazide $n=1$ ); none had any clinical evidence of other endocrine, cardiac, hepatic or renal disease. All subjects gave written informed consent and the study protocol was approved by the Research Ethics Committee of St. Vincent's Hospital.

Experimental protocol: Baseline anthropometry, body composition and habitual dietary intake were determined 1 week before starting a formula diet which continued for 4 weeks. Hyperinsulinaemic euglycaemic clamp studies were performed at baseline (day 0) and on day 4 and day 28 of the diet. Body composition was reassessed at day 28 . Subjects were weighed and reviewed by the dietitian weekly. All subjects taking oral hypoglycaemic medication discontinued therapy at least 1 week prior to commencing the study.

D iet: Each subject's diet was assessed by the dietitian on the basis of estimated 4-day food records (including weekend) using standard measures and food models for estimating portions. Energy and macronutrient intake were calculated (Diet/ 1 V3.1; Xyris Software, Brisbane, QLD, Australia) using the Nuttab 90 database of the Australian Department of Community Services and Health. Each subject was supplied with a formula diet (provided by Nutri-Metics International, Sydney, Australia). The macro-nutrient composition of the formula food per $100 \mathrm{~g}$ is: protein $37 \mathrm{~g}$, fat $2.3 \mathrm{~g}$, carbohydrate $40 \mathrm{~g}$, and energy content $1380 \mathrm{~kJ}$. The diet was customised to each subject on the basis of body size, age and energy intake to reduce energy intake by approximately $4.2 \mathrm{MJ} / \mathrm{day}$; as a result of this approach there was a wide variation between individuals in both absolute and proportional changes in macronutrient intakes (Table 1). The diet was supplemented with essential fatty acids and minerals, and free consumption of foods of negligible energy value was permitted. Subjects completed daily food records, and energy and macronutrient intakes were calculated as described above.

Body composition measurements: Body composition was assessed using a dual energy X-ray absorptiometer (DEXA; Lunar DPX - Lunar Radiation Corp., Madison, Wis., USA). Total fat mass, fat-free mass (including muscle, fluid and bone) and regional fat masses in three standard regions (trunk, arms and legs) were measured.
${ }^{\mathrm{b}}$ Significantly different from the corresponding pre-diet value $p<0.05$

A nalytical measurements: Serum leptin and serum insulin were determined using homologous radioimmunoassays (Linco, St. Charles, Mo., USA). For each subject two basal fasting samples and two samples taken at the end of a euglycaemic clamp carried out on the same day were assayed for leptin. By design mild hyperinsulinaemia was obtained during the clamps $(220 \pm 9 \mathrm{pmol} / \mathrm{l})$. Consistent with other reports [4], no effect of this mildly elevated insulin on circulating leptin was detected $(+2.7 \pm 3.7 \%$ across all studies) and therefore in subsequent analyses the leptin data were averaged across conditions (basal and hyperinsulinaemia) for each subject.

Statistical analyses were performed using the Statview and SuperAnova packages (Abacus Concepts, Berkeley, Calif., USA). Effects of energy restriction over time were assessed by one factor repeated measures analysis of variance (ANOVA). Relationships between variables were assessed using simple and multiple correlation and multiple linear regression, or ANOVA with categorised independent variables. Differences between either simple or partial correlation coefficients were assessed using Fisher's z-transformation. Differences between partial regression coefficients were assessed using a t-test. Effects of NIDDM and gender on regression relationships were assessed by analysis of covariance (ANCOVA). Data are presented as mean \pm SEM.

\section{Results and discussion}

There were significant reductions in the intakes of all macro-nutrients with an approximate $50 \%$ reduction of energy intake (Table 1). By the end of the study weight loss was $5.6 \pm 0.5 \mathrm{~kg}$ (Fig. $1 \mathrm{~A}$ ) composed of comparable amounts of total fat $(2.6 \pm 0.4 \mathrm{~kg})$ and lean body mass $(3.0 \pm 0.4 \mathrm{~kg})$, with the fat loss distributed among the regional depots (not shown). Although weight fell significantly after 4 days $(1.9 \pm 0.3 \mathrm{~kg}$, Fig. $1 \mathrm{~A})$ this largely represents fluid losses secondary to glycogen depletion and some protein loss [5]. Assuming a constant rate of fat loss over the 28 days predicts $0.3 \mathrm{~kg}$ of fat loss at day 4 .

Serum leptin concentrations were highly correlated with all measures of percent body fat derived from both DEXA and skinfold measurements on day $0 \quad(r=0.84-0.90, \quad p \leq 0.0001) \quad$ and day 28 

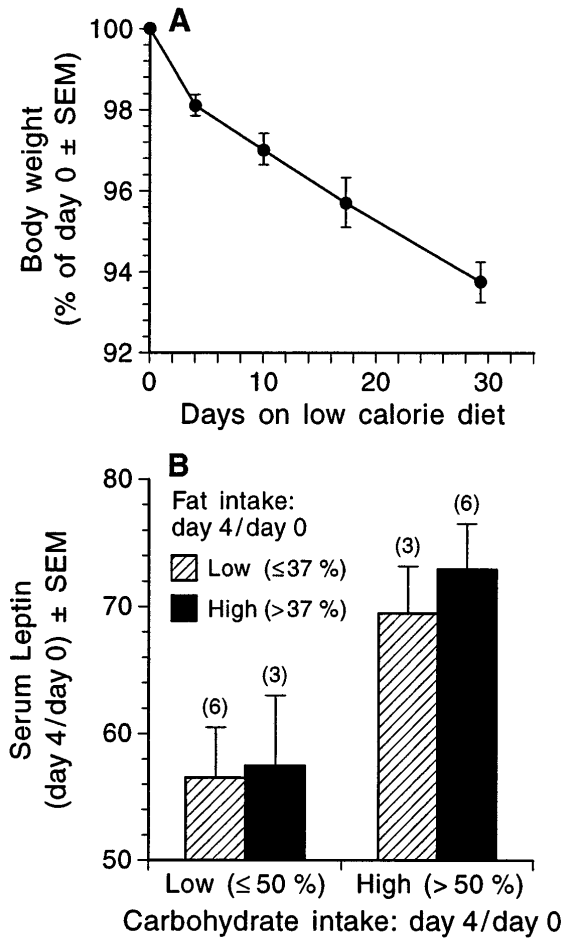

Fig. 1. A Time course of changes in body weight during energy restriction in 18 moderately obese human subjects. Data are expressed as mean \pm SEM of the percentages of the day 0 values. B Effects of changes in carbohydrate and fat intake on serum leptin after 4 days of energy restriction. All variables are expressed as the ratio day $4 /$ day 0 - the ratios for fat and carbohydrate intake were calculated from the g/day data. Macronutrient changes in each subject were categorised as high $(>$ median) or low ( $\leq$ median) and for carbohydrate averaged $55 \pm 2 \%$ and $43 \pm 1 \%$ in the high and low categories, respectively, and for fat averaged $52 \pm 4 \%$ and $29 \pm 2 \%$ in the high and low categories, respectively. Numbers in parenthesis are the numbers of subjects falling into each combination of categories. Change in carbohydrate intake is significantly associated with the change in leptin ( $p=0.012$, ANOVA) independent of changes in fat intake (main effect $p=0.65$, interaction $\mathrm{p}=0.8)$

$(r=0.69-0.77, p \leq 0.001)$. The absolute falls in serum leptin at day 4 and day 28 were strongly correlated with the day 0 value $(r=0.89, p<0.0001$ and $r=0.76, p<0.001$ respectively, not shown). Under such circumstances, analysis of absolute changes in leptin levels is not appropriate in testing for influences of other factors, and in subsequent analyses leptin levels at day 4 and day 28 were therefore expressed as percentages of the day 0 value, which did not relate significantly to the day 0 values $(r<0.4$, $p>0.1$ ). Serum leptin fell $33 \%$ by day 4 (from $19.8 \pm 2.7$ to $13.2 \pm 2.0 \mathrm{ng} / \mathrm{ml}, \mathrm{p}<0.001)$ with a further $21 \%$ fall seen at day 28 (to $9.8 \pm 1.8 \mathrm{ng} / \mathrm{ml}$ ). In simple correlations, serum leptin at day 4 (as \% of day 0 ) was significantly related to the percent change (day 4/ day $0 \%$ ) in grams of dietary carbohydrate intake per day $(r=0.74, p=0.0005)$, but not to similarly expressed changes in dietary fat $(r=0.39, p=0.11)$ or protein $(r=0.21, p=0.40)$ intakes, or to change in total energy intake (day 4/day $0 \%, r=0.44, p=0.07$ ). In a multiple correlation with the three macronutrient components, the change in carbohydrate intake was a significantly better independent correlate of the change in leptin than was the change in fat intake (partial $r=0.68$ vs $0.12, p=0.027$ ). In a multiple regression against the three macronutrient component changes (multiple $r=0.74$ ) the independent relationship between the changes in serum leptin and carbohydrate intake had a slope indistinguishable from 1 $(1.00 \pm 0.29)$, and significantly greater $(p=0.027)$ than the slope of the relationship between change in leptin and change in fat intake $(0.07 \pm 0.16)$. These relationships are illustrated in Figure $1 \mathrm{~B}$, where subjects are categorised according to the magnitude of the changes in their dietary carbohydrate and fat intakes; reflecting the correlation analysis the change in carbohydrate intake was strongly associated with the change in leptin ( $p=0.012$, ANOVA) independent of changes in fat intake (interaction $p=0.8$ ) which in isolation had no significant relationship with leptin changes $(p=0.65)$. Similar though less powerful correlations were observed at day 28 (partial $r=0.49(p=0.05), 0.33$ and 0.01 for changes in leptin vs changes in carbohydrate, fat and protein, respectively). Neither NIDDM nor gender had significant effects on the above relationships. The change in leptin at day 28 (day 28/day $0 \%$ ) was significantly related to changes in arm fat (day 28/day $4 \%$, simple $r=0.56, p=0.016$ ) but not to changes in leg, trunk or total body fat (simple $r=0.08,0.12$ and 0.39 , respectively). In a multiple regression, the loss of arm fat and the change in dietary carbohydrate were independently related to the changes in leptin levels at day 28 (regression coefficients $0.37 \pm 0.15$, and $1.09 \pm 0.41$, respectively, both $p<0.05)$ and together accounted for approximately $50 \%$ of the variance (multiple $r=0.73, p=0.003$ ).

Fasting serum insulin fell significantly at day 4 $(40 \pm 6$ vs $66 \pm 7 \mathrm{pmol} / 1, \mathrm{p}<0.0001)$ with no further change at day $28(40 \pm 8 \mathrm{pmol} / \mathrm{l})$. The early change in insulin was significantly associated with the early change in leptin levels $(r=0.57, p=0.013)$, but was not independent of change in carbohydrate intake in a multiple correlation (partial $r=0.30, p=0.22$ ). These results are consistent with a role for insulin in mediating an effect of reduced carbohydrate intake on leptin secretion. Measured whole body insulin sensitivity did not relate significantly to leptin at any time point, nor did changes in insulin sensitivity during the study relate to changes in leptin (results not shown).

These data may be interpreted as reflecting a blindness to changes in fat intake of leptin-dependent mechanisms in the short term, changes in fat intake only affecting the system in the longer term through resultant changes in body fat mass. Whether this 
acute 'fat-blindness' is a feature of normal weight regulation or is a predisposing factor for, or a concomitant of obesity cannot be answered by this study, which was restricted to obese subjects. However, there is abundant evidence from animals and humans of a failure of fat oxidation to increase acutely in response to increased dietary fat intake, eventually increasing only with increased body fat mass, in contrast to rapid stimulation of carbohydrate oxidation by increased carbohydrate intake [6]. Similarly, dietary fat appears less acutely satiating than carbohydrate [7] and, at least in mice, fat intake on a given day has a reduced effect, compared to carbohydrate, on energy intake on subsequent days [6]. The absence in the present study of a short-term relationship between leptin and dietary fat intake is consistent with this lesser satiating effect of fats and may partly explain the tendency to over-consume foods of high fat content. In summary, it is clear that the mechanisms regulating fat balance respond to changes more slowly than the mechanisms regulating carbohydrate balance. The present data suggest that the rapid response in leptin to reduced dietary carbohydrate may be a regulatory mechanism for promoting the repletion of carbohydrate stores. Consistent with this, administration of neuropeptide $\mathrm{Y}$, which is implicated in mediating leptin action, can preferentially stimulate carbohydrate intake [8].

If change in carbohydrate intake is a major determinant of leptin secretion in the short term, it seems reasonable to ask whether habitual carbohydrate intake is a determinant of circulating leptin concentration at steady state. However, whether body fat was expressed as percentage of body weight or as $\mathrm{kg}$, or carbohydrate intake was expressed as percentage of $\mathrm{kJ}$ or as g/day, no significant associations between pre-diet carbohydrate intake and leptin levels independent of body fat were found (partial correlations -0.27 to $0.27, p>0.27$ ). It may be, that in the steady state, dietary or indeed other factors which influence leptin levels in the short term have longer term influences on body fatness. Relationships between leptin and such factors would then be obscured in the steady state by the powerful dependence of leptin on the total adiposity.

The present findings may explain the relative success of the low-fat ad libitum carbohydrate diet in weight loss [9]. Reducing fat intake while maintaining or increasing carbohydrate may preserve leptin levels and hence reduce appetite and lead to better compliance. More generally, dietary or other interventions which maintain leptin levels during weight reduction may lead to improvements in weight loss.

\section{References}

1. Considine RV, Sinha MK, Heiman ML et al. (1996) Serum immunoreactive-leptin concentrations in normal weight and obese humans. New Engl J Med 334: 292-295

2. Maffei M, Halaas J, Ravussin E et al. (1995) Leptin levels in human and rodent: measurement of plasma leptin and ob RNA in obese and weight-reduced subjects. Nature Medicine 1: 1155-1161

3. MacDougald OA, Hwang CS, Fan H, Lane MD (1995) Regulated expression of the obese gene product (leptin) in white adipose tissue and 3T3-L1 adipocytes. Proc Natl Acad Sci USA 92: 9034-9037

4. Kolaczynski JW, Nyce MR, Considine RV et al. (1996) Acute and chronic effects of insulin on leptin production in humans. Diabetes 45: 699-701

5. Bray GA, Gray DS (1988) Treatment of obesity: an overview. Diabetes Metab Rev 4: 653-679

6. Flatt JP (1995) Diet, lifestyle, and weight maintenance. Am J Clin Nutr 62: 820-836

7. Blundell JE, Cotton JR, Delargy H, Green S, Greenough A, King NA, Lawton CL (1995) The fat paradox: fat-induced satiety signals versus high fat overconsumption. Int $\mathrm{J}$ Obes 19: 832-835

8. Welch CC, Grace MK, Billington CJ, Levine AS (1994) Preference and diet type affect macronutrient selection after morphine, NPY, norepinephrine, and deprivation. Am J Physiol 266: R426-R433

9. Shah M, McGovern P, French S, Baxter J (1994) Comparison of a low-fat, ad libitum complex-carbohydrate diet with a low-energy diet in moderately obese women. Am J Clin Nutr 59: 980-984 